\title{
MEASURED SECTION AND FACIES ANALYSIS OF THE LOWER CRETACEOUS FORTRESS MOUNTAIN FORMATION, ATIGUN SYNCLINE, NORTHERN ALASKA
}

\author{
by \\ Marwan A. Wartes ${ }^{1}$
}

\section{INTRODUCTION}

This study examines a thick $(>1,600 \mathrm{~m})$ section of the Lower Cretaceous Fortress Mountain Formation exposed at Atigun syncline in the foothills of the northern Brooks Range (figs. 1 and 2). To date, this interval has proven difficult to interpret (see introductory comments in Crowder, 1989); similar to other conglomerate-rich successions, limited faunal evidence and generally non-unique depositional processes hamper attempts at environmental reconstruction (e.g. Nemec and others, 1984). This ambiguity is reflected in widely varying earlier interpretations ranging from deep marine fan, to slope, to fan-delta, and even fluvial depositional settings (Crowder, 1987; 1989; Goff, 1990; Ryherd, 1990; Schenk and Bird, 1992). The purpose of this paper is to document the lithofacies and provide a composite, detailed measured section of the coarser, upper part of the formation exposed on the west face of Atigun syncline (Sheet B-1; fig. 2). This brief contribution will be followed by a more exhaustive manuscript that provides detailed lithofacies descriptions and interpretations, as well as discussion of provenance, reservoir quality, stratal stacking patterns, and fan delta classification (Wartes and Carroll, in prep.).

\section{GEOLOGIC SETTING}

The Early Cretaceous Fortress Mountain Formation (fig. 2) was deposited in the proximal reaches of the Colville foreland basin (Bird and Molenaar, 1992; Cole and others, 1997). The base is almost never exposed, but, on the basis of map relations, is usually inferred to be an angular unconformity (for example, Mull, 1985). The formation is poorly dated and plagued by limited, often long-ranging fauna. Most fossils suggest an early Albian

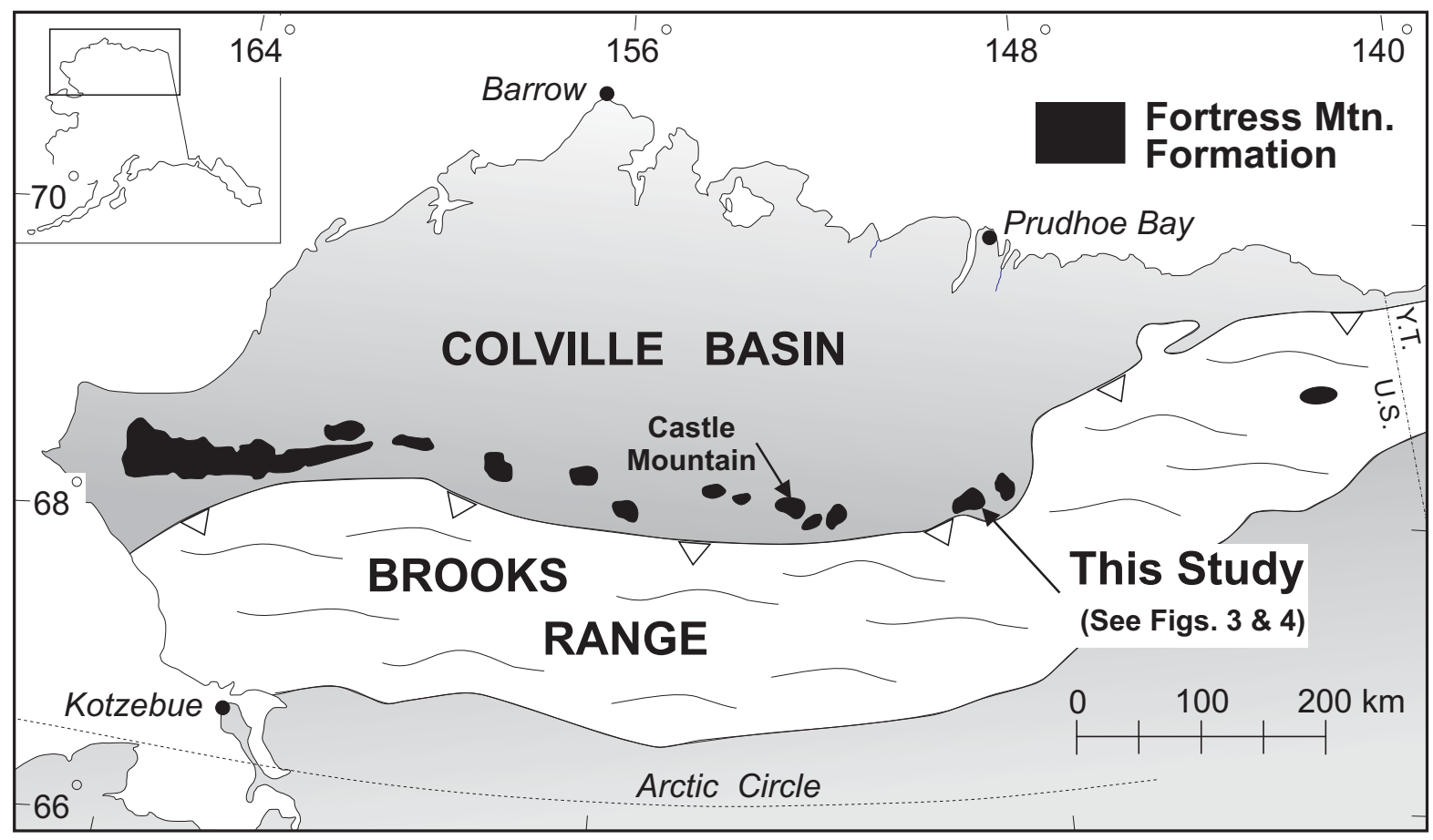

Figure 1. Distribution of principal Fortress Mountain Formation outcrops (modified from Crowder, 1987). 


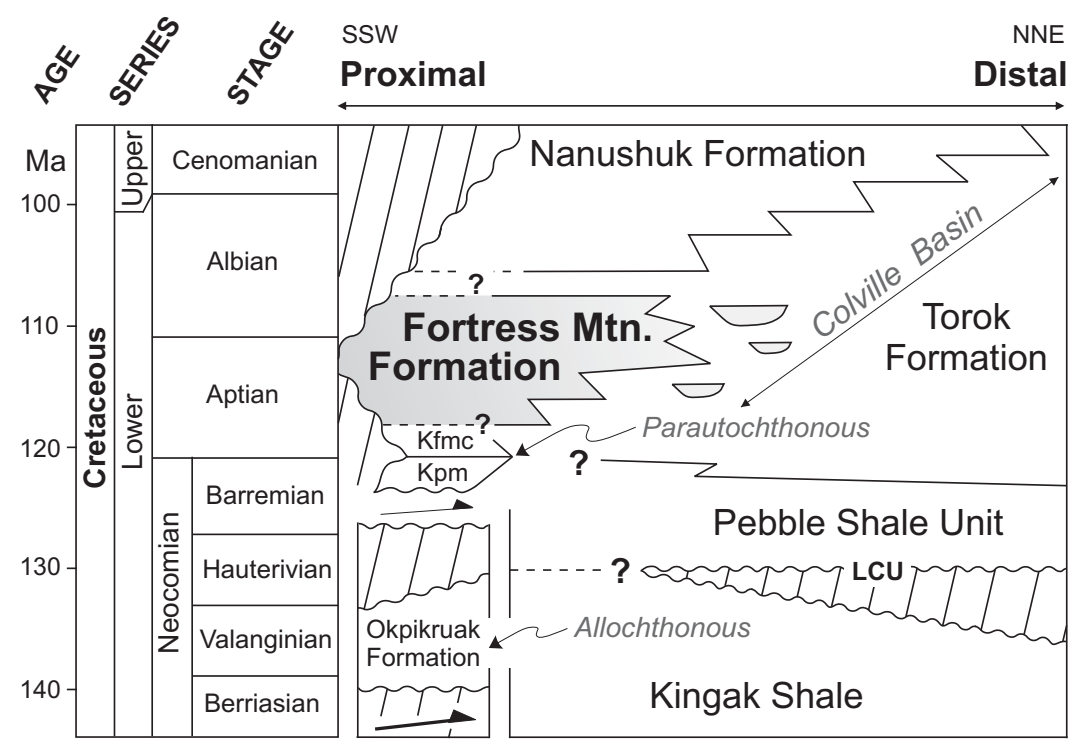

Figure 2. Chronostratigraphy of the Colville Basin, highlighting the stratigraphic and tectonic relationships between Lower Cretaceous units $(\mathrm{K} f \mathrm{mc}=$ cobblestone sandstone member of the Fortress Mountain Formation; Kpm=phosphatic-manganiferous unit; both adopted from Mull and others, 2003).

age (Molenaar and others, 1988). However, select micropaleontologic data indicate rocks as old as Hauterivian to Barremian may be present in the southern Colville Basin (fig. 2; Mickey and Haga, 1987; Mull, C.G., oral commun., 2004; Wartes, unpublished data). The type locality of the Fortress Mountain Formation has been defined at Castle Mountain (fig. 1) where an approximate thickness of 3,000 m was calculated for the type section (Patton and Tailleur, 1964).

The Fortress Mountain Formation often crops out in isolated, synclinal exposures defined by mesa-forming resistant conglomerate intervals (fig. 1). Finer-grained sandstone and siltstone facies are typically poorly exposed or covered by tundra. Rapid facies changes, especially the northward diminution of conglomerate beds, is characteristic of this formation (for example, Molenaar and others, 1988). Regionally, the Fortress Mountain Formation has been interpreted to reflect a wide array of depositional environments from basin plain to alluvial fan settings (Hunter and Fox, 1976; Crowder, 1987; Molenaar and others, 1988). The Fortress Mountain Formation grades northward into the Torok Formation, an interval characterized by poorly exposed finer-grained facies associations (Mull, 1985; fig. 2). The distinction between the Fortress Mountain and Torok Formations is somewhat arbitrary; most workers have restricted the Fortress Mountain Formation to surface exposures in the foothills of the Brooks Range (Molenaar and others, 1988).

This study focused on exposures of the Fortress Mountain Formation that crop out in Atigun syncline, a prominent open fold in the northernmost Brooks Range mountain front (figs. 3-5). Unlike most of the Brooks Range, these outcrops, lying just east of the Dalton Highway, are accessible by road (fig. 3; Mull and Harris, 1989). Previous investigations of this succession have established the general framework and basic lithofacies present (Crowder, 1987; 1989; Goff, 1990; Ryherd, 1990; Schenk and Bird, 1992; Wartes and Carroll, 2002). However, partial cover, complex facies relationships, and a lack of detailed sedimentologic analysis have resulted in considerable disagreement regarding interpreted depositional environments.

Cross sections typically depict Atigun syncline as asymmetric, with slightly steeper dips along the southern limb (for example, Mull and others, 1997). However, recent 1:63,360-scale mapping recognized more structural complexity, including local vertical dips along the north limb and compound folding in the eastern end of the syncline (Harris and others, 2002). Nevertheless, the syncline is only mildly deformed when compared with the underlying allochthonous rocks, composed in part of highly strained tectonic mélange mixed with sheared Okpikruak Formation (fig. 3; Mull and others, 1997). The basal contact of the Fortress Mountain Formation is a high-angle fault, and may have accommodated some south-vergent back-thrusting (fig. 3; Mull and others, 1997). Most authors agree that this fault is probably co-located on an angular unconformity that developed prior to deposition of the Fortress Mountain Formation (for example, Crowder, 1989).

The Fortress Mountain Formation at Atigun syncline has been informally subdivided into mappable scale lower and upper portions (figs. 3 and 6; Mull and others 1997; Harris and others, 2002). The lower Fortress Mountain Formation consists of a monotonous succession of concretionary siltstone and thin beds of very fine sandstone (figs. 6 and 7). This lower interval has also been mapped as Okpikruak Formation (Brosgé and others, 1979) and Torok Formation (Mull and Harris, 1989; Schenk and Bird, 1992). The interval exhibits locally variable deforma- 
tion, including tight folding, which may be syndepositional or reflect strain localization within this incompetent unit. The total thickness of this interval is not well constrained and complicated by an ambiguous basal contact, internal deformation, and inaccessible cliffs (fig. 7). However, this study ascribed a minimum of $450 \mathrm{~m}$ of section to the lower Fortress Mountain Formation, recognizing that a significantly thicker section is probable (fig. 6).

The upper Fortress Mountain Formation is informally defined here by the onset of thick conglomerate beds (figs. 6 and 7). This portion of the formation was studied in much greater detail in this study, a focus that is reflected by the emphasis on sandstone and conglomerate lithofacies in the subsequent facies analysis (Wartes and Carroll, 2002). During this study, approximately 1,135 m of upper Fortress Mountain Formation were measured on the west face of the syncline (figs. 5 and 6; Sheet B-1). The upper boundary of the formation is not preserved, obscuring the true depositional thickness of the unit. Previous reconnaissance studies have estimated a total formation thickness of 2,000 m (Crowder, 1987), 2,500 m (Mull and others, 1997), and 3,000 m (Crowder, 1989). This variability likely reflects different placements of the basal contact within Atigun Gorge. This study estimated approximately $1,650 \mathrm{~m}$ and is intended as a well-constrained minimum thickness for the Fortress Mountain Formation at Atigun syncline (fig. 6; Sheet B-1).

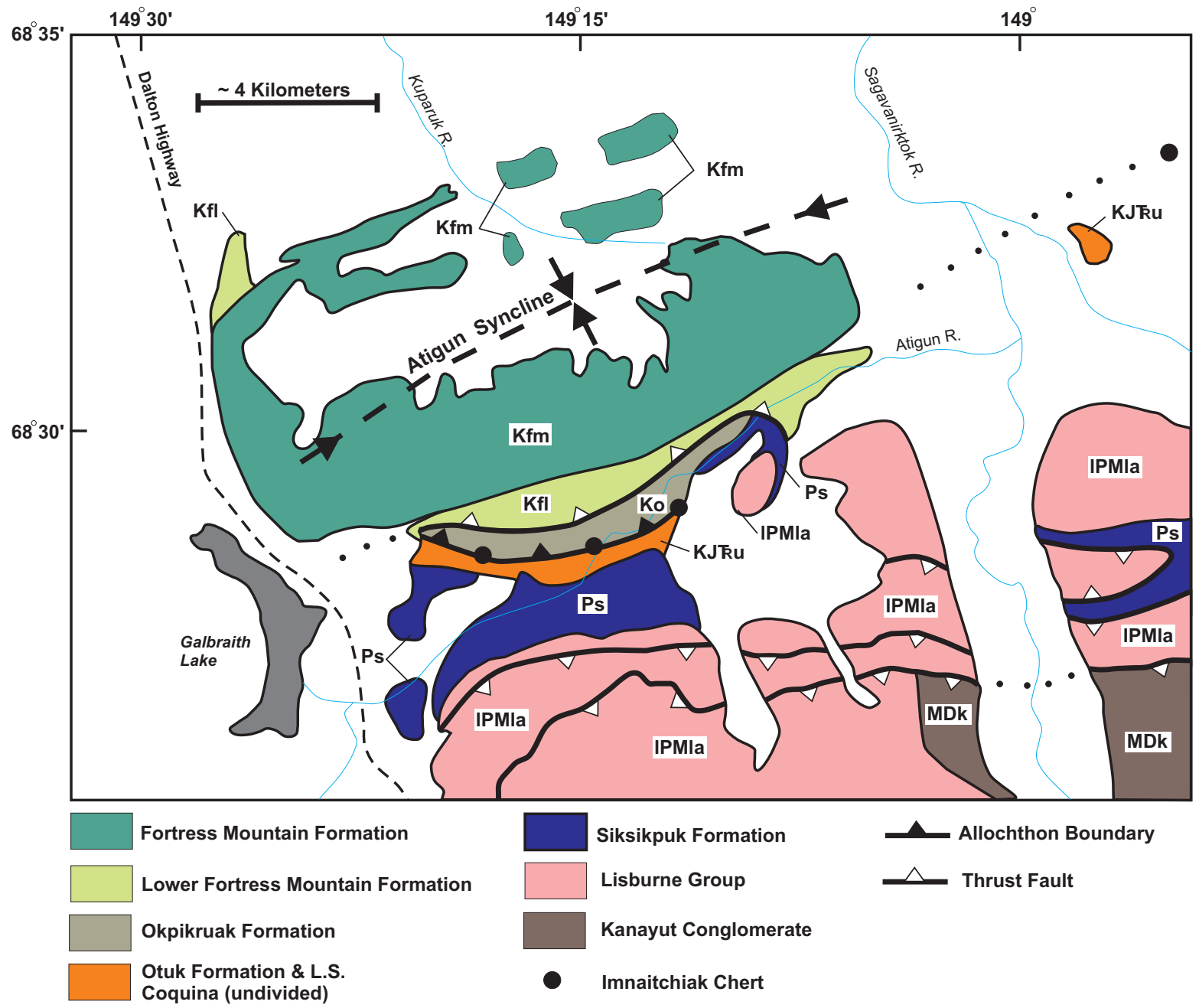

Figure 3. Simplified geologic map of the Atigun syncline area (modified from Mull and others, 1997). See Harris and others (2002) for more detailed 1:63,360-scale mapping. 


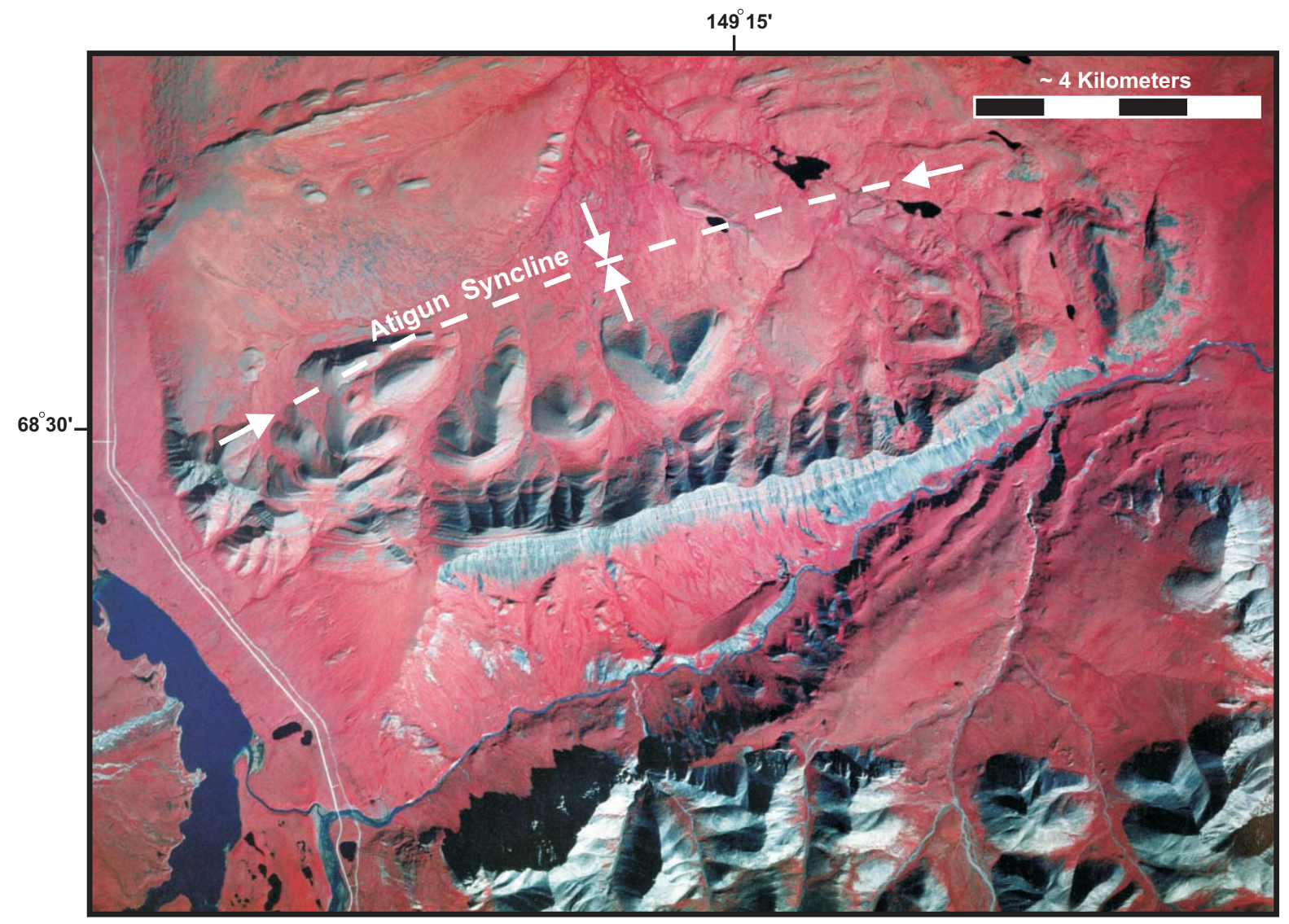

Figure 4. Air photo of the Atigun syncline area. Note the northward decrease in resistant outcrops, interpreted to reflect rapid basinward (northerly) fining of the Fortress Mountain Formation.

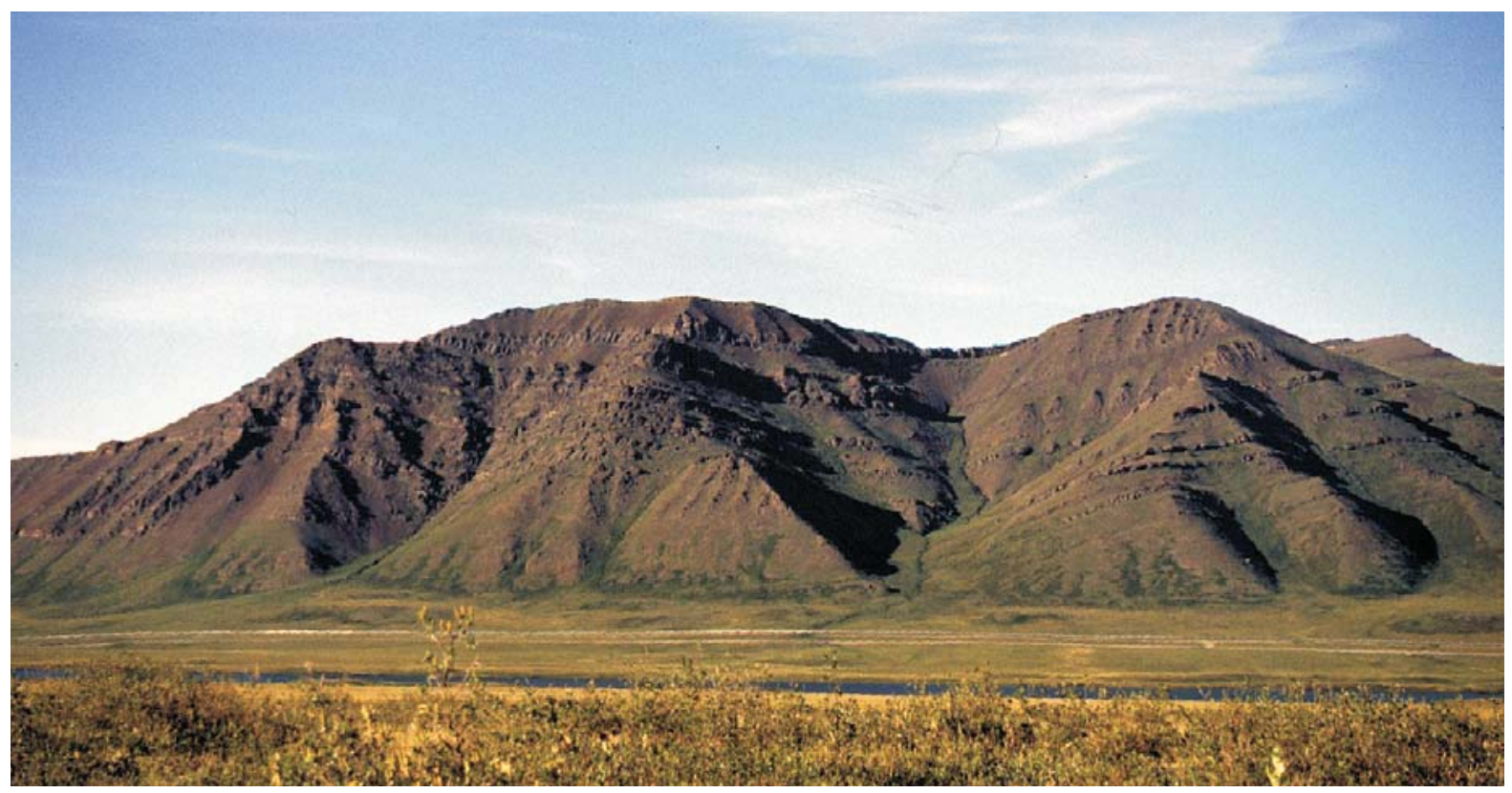

Figure 5. View of the west face of Atigun syncline where the composite section was measured (see Sheet B-1). 


\section{SEDIMENTOLOGY AND FACIES ANALYSIS}

Fan deltas form where alluvial fans prograde directly into a standing body of water (Nemec and Steel, 1988). The following section is organized around four depositional settings commonly recognized in modern and ancient fan deltas (for example, Rasmussen, 2000) and addressed below from proximal to distal: (1) alluvial, (2) delta front, (3) delta slope, and (4) prodelta. These settings interfinger laterally and often exhibit gradational contacts in vertical sections. Nevertheless, lithofacies (table 1 ) are grouped into nine unique, commonly occurring associations (table 2), which as a group suggest an environment and depositional process and provide insight into the evolution of the larger depositional system. In relating the lithofacies and facies associations in tables 1 and 2, care is taken to present observations and descriptions separate from interpretation.

Figure 6. Generalized stratigraphic column at Atigun syncline.

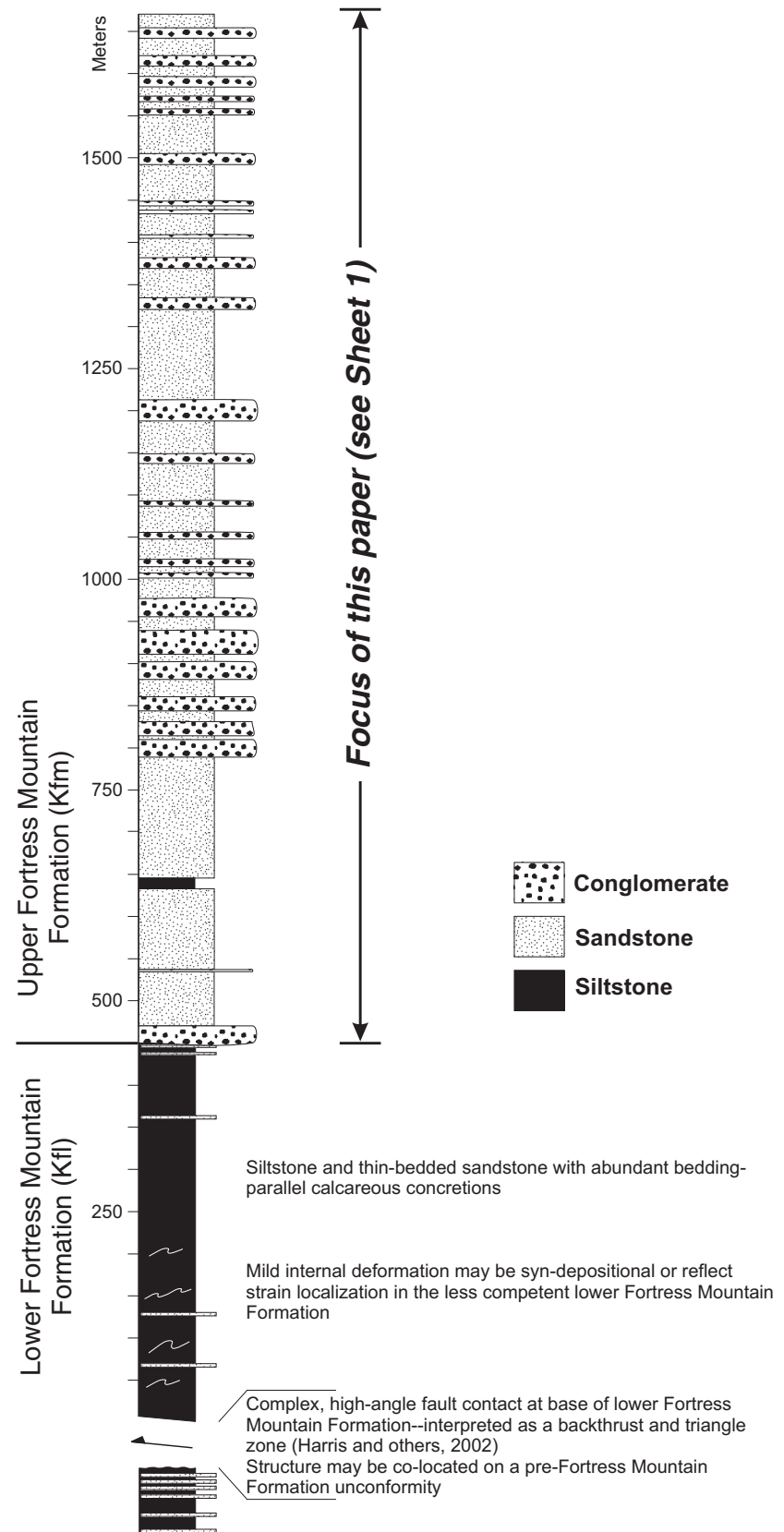

Sequence of thin-bedded turbidites--often intensely deformed into "broken formation" and structural melange (Mull and others, 1997) 


\section{Alluvial deposits}

Alluvial deposits include all recognized nonmarine environments and are collectively grouped under a single braided fluvial/alluvial fan facies association (table 2). The recognition of terminal alluvial deposits as distinct facies associations (different from genetically related subaqueous mouth bars) is an important, albeit often difficult, exercise when interpreting fan delta systems (Kleinspehn and others, 1984; Horton and Schmitt, 1996). The alluvial deposits at Atigun syncline include evidence for both confined and unconfined nonmarine depositional systems (table 1). Building on criteria established by other workers, channelized braided fluvial complexes are suggested by moderately sorted barforms and lenticular bedding (Clifton, 1973; Nemec and Steel, 1984; Hart and Plint, 2003). Paleocurrent evidence from imbricated clasts is consistent with northward progradation. Laterally persistent, unconfined alluvial fan facies are more difficult to definitively assign as nonmarine. In some cases, the designation is inferred from stratigraphic position at the top of thick upward-coarsening (and shoaling) conglomerate sequences.

Given the overall thickness of this conglomerate-rich succession, there is surprisingly sparse, unambiguous evidence for nonmarine sedimentation. An isolated coal bed was reported by Crowder (1989); however, the limited exposure does not allow one to reject a hypothesis that the bed represents a rafted allochthonous peat mat. The abundance of woody and carbonaceous debris indicates significant upstream vegetation was incorporated, probably from cannibalization of the delta plain. Although no paleosols or related diagnostic facies were observed in this study, it remains possible that delta plain deposits (such as overbank fine-grained facies) exist under

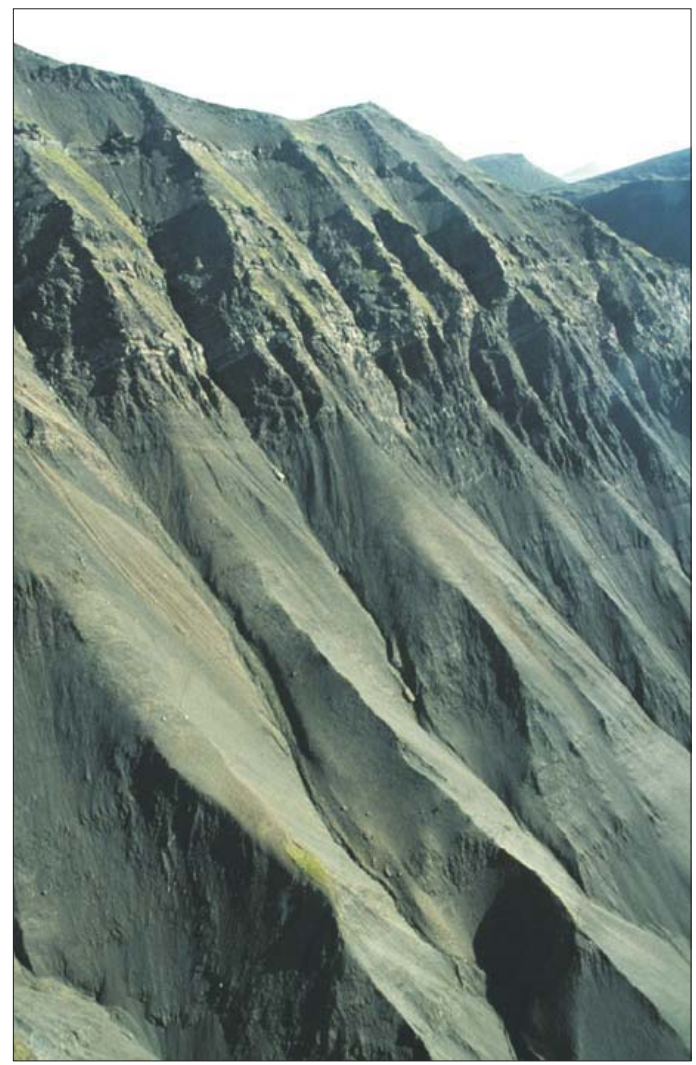

Figure 7. View of steep cliff exposures of the finegrained lower Fortress Mountain Formation in Atigun Gorge. some covered intervals.

Table 1. Description and interpretation of lithofacies (codes modified and extended from Miall [1978])

\begin{tabular}{|c|c|c|c|}
\hline Lithofacies & Code & Description & Interpretation \\
\hline & \multicolumn{3}{|c|}{ Conglomerate } \\
\hline $\begin{array}{l}\text { Massive clast- } \\
\text { supported } \\
\text { conglomerate }\end{array}$ & $\mathrm{Gm}$ & $\begin{array}{l}\text { Poorly sorted, massive to weakly stratified, } \\
\text { ungraded granule-pebble conglomerate; } \\
\text { often amalgamated; rare weak imbrication }\end{array}$ & $\begin{array}{l}\text { Cohesionless debris flow, } \\
\text { hyperconcentrated flow, and gravelly high- } \\
\text { density turbidity current }\end{array}$ \\
\hline $\begin{array}{l}\text { Graded clast- } \\
\text { supported } \\
\text { conglomerate }\end{array}$ & Gng & $\begin{array}{l}\text { Poorly sorted, granule-pebble conglomerate; } \\
\text { commonly grading into pebbly sandstone }\end{array}$ & $\begin{array}{l}\text { Rapid deposition of waning high- } \\
\text { concentration flow (floods) and gravelly } \\
\text { high-density turbidity current }\end{array}$ \\
\hline $\begin{array}{l}\text { Inversely graded } \\
\text { conglomerate }\end{array}$ & Gin & $\begin{array}{l}\text { Poorly sorted, granule-pebble conglomerate; } \\
\text { weak inverse grading in upper part of beds; } \\
\text { irregular bed tops with protruding clasts }\end{array}$ & Rapid freezing of cohesive debris flow \\
\hline $\begin{array}{l}\text { Matrix- } \\
\text { supported } \\
\text { conglomerate }\end{array}$ & Gmm & $\begin{array}{l}\text { Chaotic to massive, poorly sorted, matrix- } \\
\text { supported pebble to boulder conglomerate; } \\
\text { often subangular clasts }\end{array}$ & $\begin{array}{l}\text { Subaerial debris flow and } \\
\text { hyperconcentrated flow }\end{array}$ \\
\hline $\begin{array}{l}\text { Open-framework } \\
\text { conglomerate }\end{array}$ & Gof & $\begin{array}{l}\text { Well sorted, open-framework, granule-- } \\
\text { pebble conglomerate; bi-directional } \\
\text { imbrication of disk-shaped clasts }\end{array}$ & $\begin{array}{l}\text { Strong reworking and winnowing during } \\
\text { wave run-up; bedload deposition of } \\
\text { turbulent high-energy flow }\end{array}$ \\
\hline $\begin{array}{l}\text { Trough cross- } \\
\text { stratified } \\
\text { conglomerate }\end{array}$ & Gt & $\begin{array}{l}\text { Trough cross-stratified conglomerate; clast- } \\
\text { supported, moderate sorting }\end{array}$ & $\begin{array}{l}\text { Bedload traction current; migration of } \\
\text { sinuous crested gravel bedforms }\end{array}$ \\
\hline
\end{tabular}


Table 1. Description and interpretation of lithofacies (codes modified and extended from Miall [1978])continued

\begin{tabular}{|c|c|c|c|}
\hline Lithofacies & Code & Description & Interpretation \\
\hline & \multicolumn{3}{|c|}{ Conglomerate (continued) } \\
\hline $\begin{array}{l}\text { Mega-rippled } \\
\text { granule } \\
\text { conglomerate }\end{array}$ & GSr & $\begin{array}{l}\text { Mega-rippled granule conglomerate to very } \\
\text { coarse sandstone; poor to moderate sorting; } \\
\text { straight crested with } \sim 50 \mathrm{~cm} \text { wavelength and } \\
\sim 15 \mathrm{~cm} \text { amplitude }\end{array}$ & $\begin{array}{l}\text { Unidirectional and oscillatory bedload } \\
\text { traction current; migration of linear crested } \\
\text { bedforms }\end{array}$ \\
\hline $\begin{array}{l}\text { Conglomerate } \\
\text { lag }\end{array}$ & Glg & $\begin{array}{l}\text { Granule to very coarse sandstone stringers } \\
\text { and trains; } 1-5 \text { grains thick; horizontally } \\
\text { bedded or broadly lining low-angle bedforms }\end{array}$ & $\begin{array}{l}\text { Traction flow; size segregation due to } \\
\text { winnowing during barform migration or the } \\
\text { generation of low-relief scours }\end{array}$ \\
\hline Scour fill & Gsc & $\begin{array}{l}\text { Lens-shaped conglomerate beds with } \\
\text { irregular basal contact; rare weak imbrication }\end{array}$ & $\begin{array}{l}\text { Channel-form incision and infill of high- } \\
\text { concentration turbulent gravel flow }\end{array}$ \\
\hline
\end{tabular}

\begin{tabular}{|c|c|c|c|}
\hline \multirow[b]{2}{*}{$\begin{array}{l}\text { Planar-laminated } \\
\text { sandstone }\end{array}$} & \multicolumn{3}{|c|}{ Sandstone } \\
\hline & Sh & $\begin{array}{l}\text { Planar-laminated to thinly-bedded sandstone; } \\
\text { dominantly fine-grained }\end{array}$ & $\begin{array}{l}\text { Lower and upper flow regime planar flow; } \\
\text { tractive transport }\end{array}$ \\
\hline $\begin{array}{l}\text { Trough cross- } \\
\text { stratified } \\
\text { sandstone }\end{array}$ & St & $\begin{array}{l}\text { Cross-stratified sandstone; dominantly } \\
\text { trough, rare planar tabular geometry; thin to } \\
\text { thick bedded; occasional floating granules; } \\
\text { marine bivalves, occasionally in coquina } \\
\text { beds }\end{array}$ & $\begin{array}{l}\text { Traction current deposition from migration } \\
\text { of sinuous-crested (3-D) dune bedforms; } \\
\text { mid to upper part of lower flow regime }\end{array}$ \\
\hline Scour fill & Ssc & $\begin{array}{l}\text { Lenticular sandstone bed with irregular basal } \\
\text { contact }\end{array}$ & Erosive incision and subsequent infill \\
\hline $\begin{array}{l}\text { Steep sandstone } \\
\text { foresets }\end{array}$ & Scg & $\begin{array}{l}\text { Steeply inclined sandstone foresets }\left(15^{\circ}-\right. \\
\left.35^{\circ}\right) ; 40-50-\mathrm{cm} \text {-thick beds; occasional } \\
\text { internal grading }\end{array}$ & $\begin{array}{l}\text { Slipface deposition of large 2-D migrating } \\
\text { sand ridges or barforms }\end{array}$ \\
\hline $\begin{array}{l}\text { Rippled } \\
\text { sandstone }\end{array}$ & $\mathrm{Sr}_{1}$ & $\begin{array}{l}\text { Rippled sandstone; straight to sinuous } \\
\text { crested; rare interference pattern; } \\
\text { occasionally bioturbated }\end{array}$ & $\begin{array}{l}\text { Traction flow and migration of ripple } \\
\text { bedforms; low-flow regime }\end{array}$ \\
\hline $\begin{array}{l}\text { Mega-rippled } \\
\text { sandstone }\end{array}$ & $\mathrm{Sr}_{2}$ & $\begin{array}{l}\text { Mega-rippled coarse- to very-coarse-grained } \\
\text { sandstone; moderate sorting; straight crested } \\
\text { with } \sim 50 \mathrm{~cm} \text { wavelength and } \sim 15 \mathrm{~cm} \\
\text { amplitude }\end{array}$ & $\begin{array}{l}\text { Unidirectional and oscillatory bedload } \\
\text { traction current; migration of straight- } \\
\text { crested bedforms }\end{array}$ \\
\hline $\begin{array}{l}\text { Low-angle } \\
\text { cross-stratified } \\
\text { sandstone }\end{array}$ & Sl & $\begin{array}{l}\text { Thin-bedded, low-angle, cross-stratified } \\
\text { sandstone; occasionally laminated; rare } \\
\text { floating granules }\end{array}$ & $\begin{array}{l}\text { Bedload traction modified by long period } \\
\text { oscillatory(?) current }\end{array}$ \\
\hline $\begin{array}{l}\text { Massive poorly } \\
\text { sorted sandstone }\end{array}$ & $\mathrm{Sm}$ & $\begin{array}{l}\text { Massive, fine-grained sandstone with } \\
\text { abundant floating, very-coarse sandstone } \\
\text { grains and granules; occasional weak } \\
\text { grading; clasts oriented randomly or more } \\
\text { rarely in pockets; occasional unstable clast } \\
\text { orientations; scattered marine bivalves }\end{array}$ & $\begin{array}{l}\text { Sandy debris flows, hyperconcentrated } \\
\text { flows, and high-density turbidity currents; } \\
\text { rapid freezing of a traction carpet }\end{array}$ \\
\hline $\begin{array}{l}\text { Hummocky } \\
\text { cross-stratified } \\
\text { sandstone }\end{array}$ & Shc & $\begin{array}{l}\text { Hummocky cross-stratified sandstone; very } \\
\text { fine grained; bedform relief of } \sim 20 \mathrm{~cm}\end{array}$ & $\begin{array}{l}\text { Long period oscillatory-dominated } \\
\text { combined flow }\end{array}$ \\
\hline \multirow[t]{2}{*}{$\begin{array}{l}\text { Sandstone- } \\
\text { Mudstone } \\
\text { couplets }\end{array}$} & SM & $\begin{array}{l}\text { Thin-bedded couplets of graded medium- to } \\
\text { fine-grained sandstone and siltstone (various } \\
\text { Bouma sequences); mudstone rip-ups; } \\
\text { carbonaceous debris; ripples; bioturbation }\end{array}$ & $\begin{array}{l}\text { Low- to high-density turbidite currents and } \\
\text { hyperpycnal flows }\end{array}$ \\
\hline & \multicolumn{3}{|c|}{ Fine-grained deposits } \\
\hline $\begin{array}{l}\text { Massive } \\
\text { siltstone }\end{array}$ & Fml & Massive to weakly planar-laminated siltstone & Suspension deposits \\
\hline $\begin{array}{l}\text { Ripple- } \\
\text { laminated } \\
\text { siltstone }\end{array}$ & $\mathrm{Fr}$ & $\begin{array}{l}\text { Weakly graded very-fine-grained sandstone } \\
\text { to siltstone; commonly well rippled; } \\
\text { occasionally calcareous alteration of } \\
\text { sandstone }\end{array}$ & $\begin{array}{l}\text { Low-density, dilute turbidite currents and } \\
\text { hyperpycnal flows }\end{array}$ \\
\hline $\begin{array}{l}\text { Bioturbated } \\
\text { siltstone }\end{array}$ & $\mathrm{Fb}$ & $\begin{array}{l}\text { Massive siltstone with vertical burrows and } \\
\text { occasional horizontal feeding traces }\end{array}$ & Bioturbated deposits \\
\hline Coal & $\mathrm{C}$ & $\begin{array}{l}\text { Massive coal; } \sim 75 \mathrm{~cm} \text { thick; limited lateral } \\
\text { extent }\end{array}$ & $\begin{array}{l}\text { Concentration of organic matter in quiet } \\
\text { setting }\end{array}$ \\
\hline
\end{tabular}


Table 2. Summary of facies associations

\begin{tabular}{|c|c|c|c|c|}
\hline $\begin{array}{l}\text { Depositional } \\
\text { Setting }\end{array}$ & $\begin{array}{c}\text { Facies } \\
\text { Association }\end{array}$ & Lithofacies & Description and Occurrence & Interpretation \\
\hline \multirow[t]{2}{*}{ Alluvial } & Alluvial Fan & $\begin{array}{l}\text { (Gm, Gng, } \\
\text { Gin, Gmm, } \\
\text { Gsc, Ssc) }\end{array}$ & $\begin{array}{l}\text { Massive to weakly stratified } \\
\text { conglomerate; dominantly matrix- } \\
\text { supported fabrics; rare thin sandstone } \\
\text { beds; up to tens of meters thick; best } \\
\text { observed at Hoary Marmot Mountain }\end{array}$ & $\begin{array}{l}\text { Subareal debris flow and } \\
\text { sheet flood events in a } \\
\text { proximal alluvial fan }\end{array}$ \\
\hline & Delta Plain & $\begin{array}{l}\text { (Gm, Gng, } \\
\text { Gt, Glg, } \\
\text { Gsc, Sh, St, } \\
\left.\text { Ssc, } \mathrm{Sr}_{1}, \mathrm{C}\right)\end{array}$ & $\begin{array}{l}\text { Horizontally stratified conglomerate and } \\
\text { lesser sandstone; rare broad channel- } \\
\text { form geometry; occurs at the top of } \\
\text { coarsening-upward sequences; } \\
\text { intimately associated with the mouth bar } \\
\text { facies association }\end{array}$ & $\begin{array}{l}\text { Gravel-dominated, braided } \\
\text { fluvial system characterized } \\
\text { by flood stage processes; } \\
\text { limited preservation of } \\
\text { overbank facies }\end{array}$ \\
\hline \multirow[t]{4}{*}{$\begin{array}{l}\text { Fan Delta } \\
\text { Front }\end{array}$} & Mouth Bar & $\begin{array}{l}\text { (Gm, Gng, } \\
\text { Gin, Gt, } \\
\text { Gr, Glg, } \\
\text { Gsc, Sh, St, } \\
\mathrm{Ssc}, \mathrm{Sr}_{1}, \\
\mathrm{Sr}_{2}, \mathrm{Sm}, \\
\mathrm{Scg}(?))\end{array}$ & $\begin{array}{l}\text { Planar-bedded, sheet-like intervals of } \\
\text { coarsening-upward conglomerate with } \\
\text { minor sandstone intervals; often forms a } \\
\text { relatively sharp contact Mewith } \\
\text { underlying fine-grained sandstone of the } \\
\text { distal mouth bar and shoreface facies } \\
\text { associations }\end{array}$ & $\begin{array}{l}\text { Progradation of river- } \\
\text { dominated mouth bars into a } \\
\text { shallow marine setting; rapid } \\
\text { deposition (flood events) of } \\
\text { sediment-laden flows; } \\
\text { variable reworking by wave } \\
\text { processes }\end{array}$ \\
\hline & Beachface & $\begin{array}{l}\text { (Gof, Gr, } \\
\text { Sh, Sr1, } \\
\text { Sr2) }\end{array}$ & $\begin{array}{l}\text { Thin, distinct zones of well sorted } \\
\text { conglomerate and sandstone; beds are } \\
\text { laterally discontinuous and grade into } \\
\text { poorly sorted lithofacies; where present, } \\
\text { usually occur at the very top of } \\
\text { coarsening-upward trends }\end{array}$ & $\begin{array}{l}\text { Intense winnowing during } \\
\text { wave run-up; probable } \\
\text { reworking of previously } \\
\text { deposited mouth bar gravels }\end{array}$ \\
\hline & $\begin{array}{l}\text { Distal Mouth } \\
\text { Bar }\end{array}$ & $\begin{array}{l}\text { (Gm, Gng, } \\
\text { Gsc, St, } \\
\text { Ssc, Sm, } \\
\text { Fml) }\end{array}$ & $\begin{array}{l}\text { Massive, pebbly sandstone and lesser } \\
\text { conglomerate; laterally continuous } \\
\text { geometry; intercalated with the } \\
\text { shoreface facies association and } \\
\text { commonly grades upward into mouth } \\
\text { bar conglomerate }\end{array}$ & $\begin{array}{l}\text { Flood-influenced sandy } \\
\text { debris-flow deposition; } \\
\text { offshore of active sites of } \\
\text { mouth bar lobe progradation }\end{array}$ \\
\hline & Shoreface & $\begin{array}{l}\text { (Gt, Gr, } \\
\text { Glg, Sh, St, } \\
\mathrm{Sr}_{1}, \mathrm{Sr}_{2}, \mathrm{Sl} \\
\mathrm{Scg}(?) \\
\text { Fml, Fb) }\end{array}$ & $\begin{array}{l}\text { Thin-bedded or laminated sandstone; } \\
\text { usually cross-stratified; interbedded } \\
\text { with distal mouth bar deposits }\end{array}$ & $\begin{array}{l}\text { Discontinuous influence of } \\
\text { wave processes (storm?); } \\
\text { located off-axis of active } \\
\text { mouth bar settings }\end{array}$ \\
\hline \multirow[t]{3}{*}{$\begin{array}{l}\text { Fan Delta } \\
\text { Slope }\end{array}$} & Turbidite & $\begin{array}{l}\text { (Sh, } \mathrm{Sr}_{1}, \\
\mathrm{SM}, \mathrm{Fml})\end{array}$ & $\begin{array}{l}\text { Very-fine-grained sandstone separated } \\
\text { by siltstone drapes; tabular and laterally } \\
\text { continuous; coarsen and thicken upward } \\
\text { into distal mouth bar or shoreface facies } \\
\text { associations }\end{array}$ & $\begin{array}{l}\text { Pelagic silt deposition, } \\
\text { punctuated by sandy } \\
\text { turbidites and possible } \\
\text { hyperpycnal flows }\end{array}$ \\
\hline & Storm & (St, Sl Shc) & $\begin{array}{l}\text { Fine- to very-fine-grained sandstone; } \\
\text { usually laminated and characterized by } \\
\text { low-angle cross-stratification; } \\
\text { interstratified with the turbidite facies } \\
\text { association }\end{array}$ & $\begin{array}{l}\text { Combined oscillatory and } \\
\text { unidirectional flow set up by } \\
\text { large storm wave orbitals }\end{array}$ \\
\hline & Channel & $\begin{array}{l}\text { (Gm, Gng, } \\
\text { Sm, Ssc) }\end{array}$ & $\begin{array}{l}\text { Sharp-based channel-forms, } \\
\text { approximately } 10 \text { m deep, and filled } \\
\text { with flat-lying conglomerate fining- } \\
\text { upward to sandstone; conglomerate } \\
\text { includes rip-ups of underlying mudstone }\end{array}$ & $\begin{array}{l}\text { Incision of submarine } \\
\text { channel, possibly during } \\
\text { base level fall; subsequent } \\
\text { bypass and eventual infilling } \\
\text { of pre-existing sediment } \\
\text { conduit }\end{array}$ \\
\hline Prodelta & Prodelta & $\begin{array}{l}\text { (Fml, Fr, } \\
\text { Fb) }\end{array}$ & $\begin{array}{l}\text { Continuous sheet-like deposits, defined } \\
\text { by calcareous very-fine-grained } \\
\text { sandstone horizons; ripple trains } \\
\text { common, bioturbation present; } \\
\text { characterizes several hundred meters of } \\
\text { continuous section in the lower Fortress } \\
\text { Mountain Formation }\end{array}$ & $\begin{array}{l}\text { Pelagic deposition with } \\
\text { minor low-density silt- } \\
\text { dominated turbidites }\end{array}$ \\
\hline
\end{tabular}




\section{Fan delta front deposits}

The fan delta front marks the subaqueous nearshore setting characterized by the interplay of riverine (flood-dominated) and marine (wave-dominated) processes (Kleinspehn and others, 1984; Rasmussen, 2000). The four principal facies associations include: (1) proximal mouth bar, (2) beachface, (3) distal mouth bar, and (4) shoreface (table 2). The fan delta front is a natural trap for gravel as both stream- and mass flow-dominated alluvial systems decelerate upon entering a standing body of water (for example, Nemec and Steel, 1984). The best exposures at Atigun syncline occur in conglomeratic fan delta front deposits which dominate the southernmost coarse-grained exposures (fig. 5). The component facies associations reflect the interaction of flood-dominated and marineinfluenced processes (tables 1 and 2). However, it should be noted that many of these deposits are closely interbedded and certainly existed lateral to one another at any given time. The proximal mouth bar facies association is characterized by massive, poorly sorted mass-flow deposits that rapidly fine northward into distal mouth bar facies association (fig. 8). The subaqueous nature of these deposits is confirmed by occasional marine bivalves and probable wave-related megaripples (fig. 9; Sheet B-1).

The shoreface facies association is often poorly exposed and interpreted to underlie extensive tundra-covered intervals along the southern limb of the syncline. This association is dominated by cross-stratified sandstone (fig. 9) and records episodes of wave reworking of the fan delta front. This association is often closely interbedded with distal mouth bar deposits, suggesting a competition between sediment delivery during flood events and marine reworking by waves. However, the limited conglomerate may suggest that shoreface deposits are best developed (and preserved) in inactive sectors of the fan delta, possibly removed from sites of active sediment influx. The beachface facies association records a key zone where wave run-up produces thin accumulations of strongly winnowed, openframework gravel (fig. 9). Beachface deposits, where present, occur in the upper portion of conglomerate-dominated packages (Sheet B1 ), and record the precise shoreline position. Similar to the shoreface facies association, the preservation of wave-reworked beach deposits probably reflects sites on the fan delta front not actively receiving land-derived sediment (Lopez-Blanco, 1993).
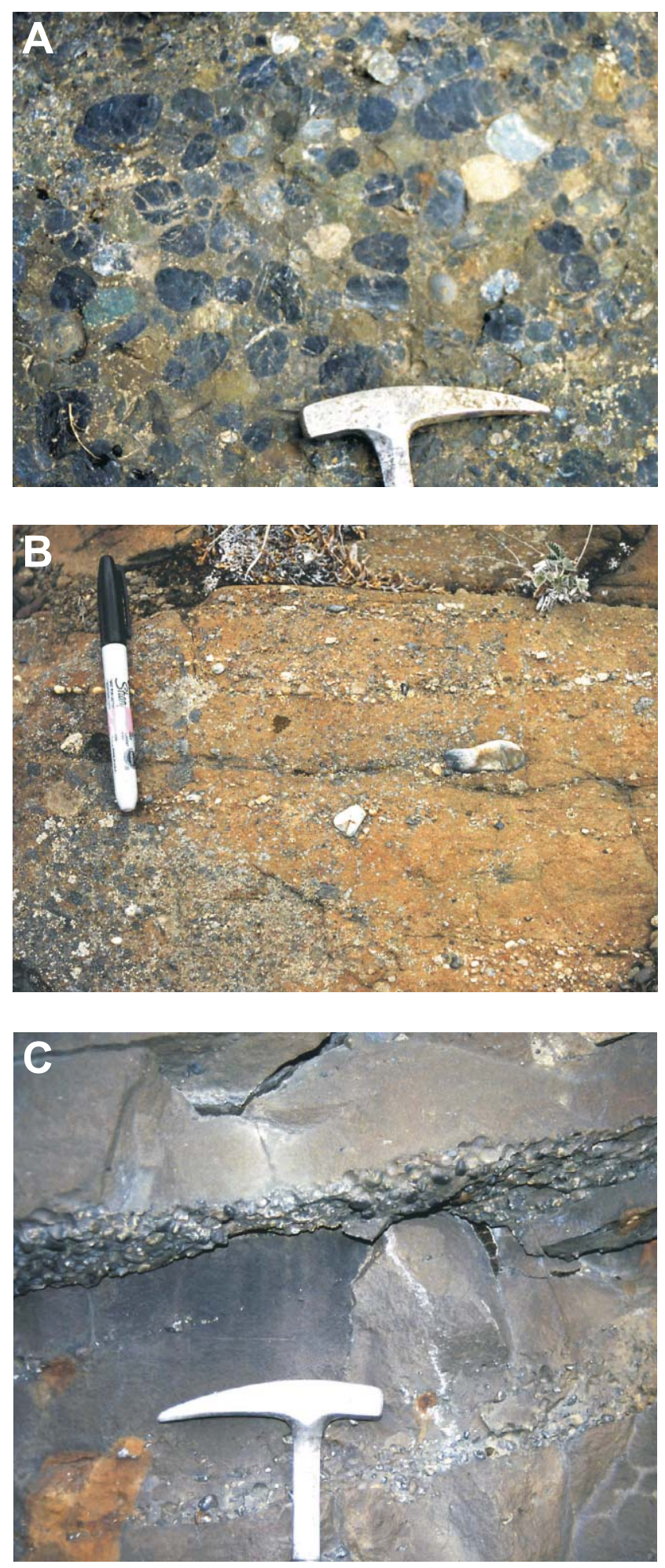

Figure 8. Photos of proximal and distal mouth bar facies associations. (A) Typical texture within matrix-rich, massive pebble conglomerate. (B) Poorly sorted fine-grained sandstone with floating granules and pebbles common to the distal mouth bar facies association. (C) Discontinuous coarse sandstone to granule lags in fine-grained sandstone. 

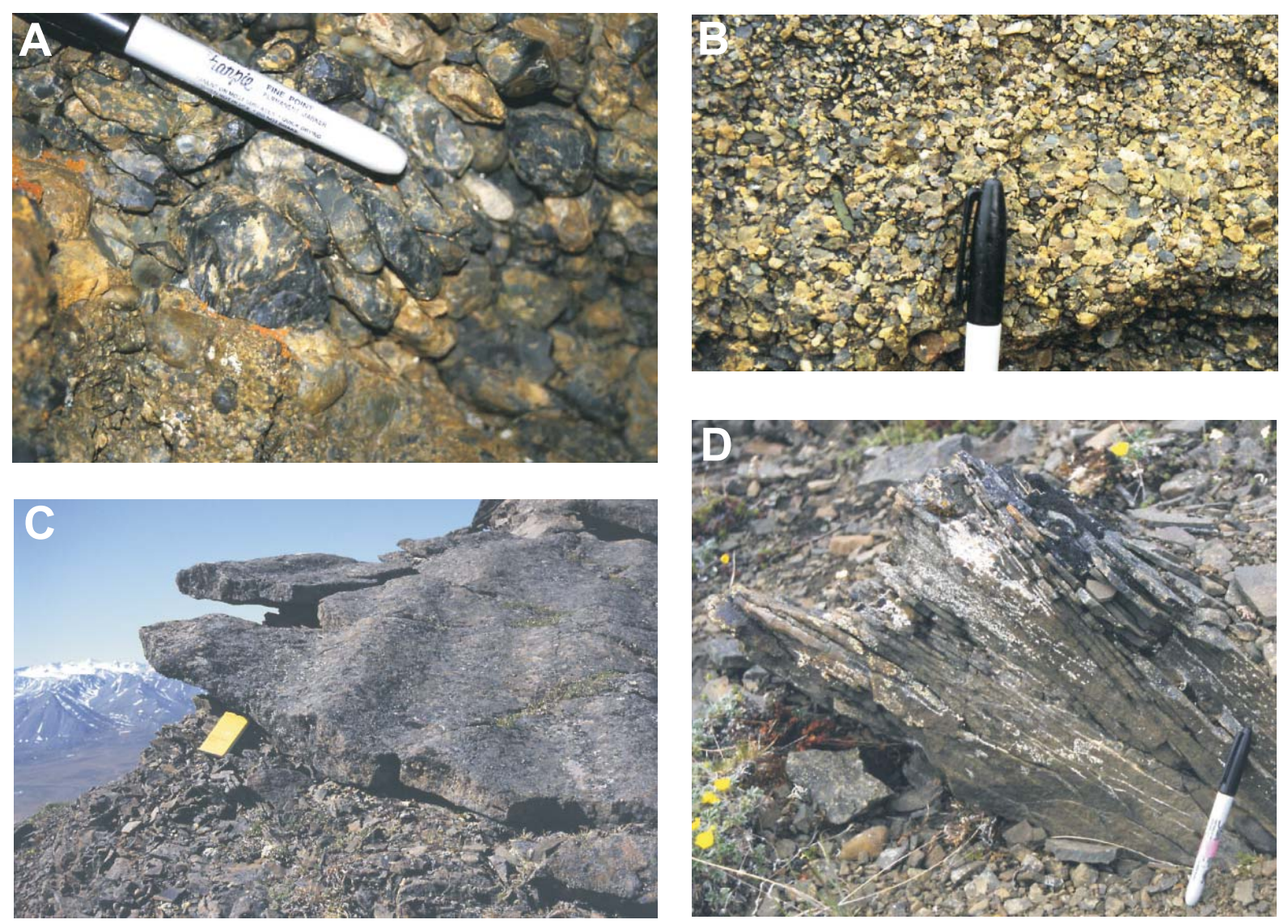

Figure 9. Photos of wave-influenced sedimentary structures within the fan delta front. (A) Example of well developed openframework textures in foreshore gravel. The majority of clasts are north dipping, suggesting imbrication during wave run-up. (B) Moderate to well sorted granule conglomerate interpreted as wave-winnowed foreshore facies. (C) Straight crested megaripples formed by wave reworking of mouth bar deposits; sense of transport is to the south. (D) Thin-bedded, cross-stratified sandstone typical of the shoreface facies association.

\section{Fan delta slope deposits}

The fan delta slope setting (in the sense of Postma, 1984) lies basinward of the fan delta front and below wave base (excepting large storms). The delta slope is here subdivided into three component facies associations: (1) turbidite, (2) storm, and (3) channel/chute (table 2). Fan delta slope deposits at Atigun syncline tend to be conglomerate-poor, leading to limited exposure. The three recognized facies associations reflect a spectrum of processes including: delta-fed turbidity currents (fig. 10), hyperpycnal flows, periodic storm reworking, and the incision and backfilling of small channels. Fan delta slope deposits are locally observed along the southwest corner of the fold where they mark the lower portions of large upward-coarsening packages that shoal upward into proximal fan delta front deposits. It remains possible that fan delta slope facies, particularly the turbidite association, exist beneath covered swales between resistant conglomerate beds (Sheet B-1). Nevertheless, this environment is best preserved in select stream cuts in the central and northern portions of the syncline and is suspected to underlie much of the tundra-covered regions (figs. 3 and 4). The increased abundance of fan delta slope facies to the north is consistent with the interpreted northward deepening of the basin.

\section{Prodelta deposits}

The prodelta environment lies basinward of the fan delta slope. Similar to normal deltas, the prodelta setting is typically dominated by fine-grained deposits and represents the most distal component of a deltaic prism. Prodelta deposits are largely confined to the fine-grained lower Fortress Mountain Formation, which was not examined in detail during this study (generalized in fig. 6). This monotonous sequence of siltstone and very fine sandstone records the most distal component of this depositional system. The lower Fortress Mountain Formation is best 

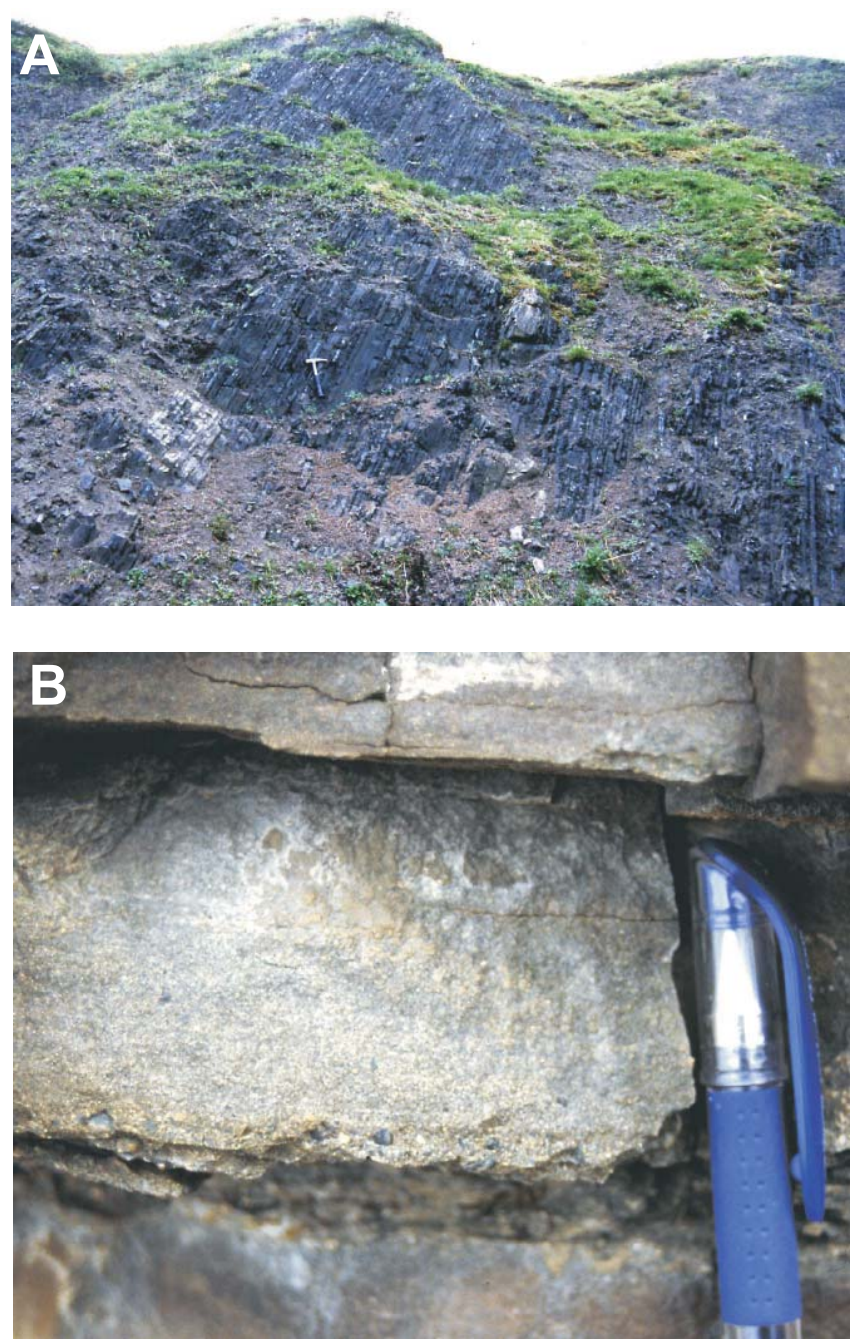

Figure 10. Photos of fan delta slope facies. (A) Rhythmic, very finegrained sandstone-dominated turbidites. (B) Example of well preserved Bouma A and B subdivisions. displayed in steep cliff exposures on the southern limb of the fold (fig. 7), although prodelta deposits are also observed in a small quarry in the northwest limb of the fold (figs. 3, 4, and 6).

\section{DISCUSSION AND CONCLUSIONS}

The body of evidence in the preceding analysis and outlined in tables 1 and 2 supports the interpretation that Atigun syncline was the site of a long-lived fan deltaic depocenter along the ancestral mountain front of the Brooks Range orogen. The distribution of the nine facies associations is schematically reconstructed in figure 11 . This fan delta model best reconciles the close association of wave, fluvial, and mass flow processes recognized in this study. The key observations constraining this interpretation stem from the new recognition of wave-related associations, especially the mega-rippled surfaces and imbricated foreshore gravels (fig. 9). These important environmental interpretations are bolstered by wave-generated, landward-directed paleocurrents that effectively limit the water depth to well within fair-weather wave base. As noted by many authors, knowledge of the shoreline position is a key stratigraphic marker in fan deltas (Wescott and Ethridge, 1980; Bourgeois and Leithold, 1984; Massari and Parea, 1988; Horton and Schmitt, 1996).

Paleocurrents, such as large proximal mouth bar foresets, indicate a dominantly northward direction of progradation (fig. 12). A northward deepening of the basin is further indicated by the abrupt loss of conglomerate beds to the north (fig. 4). This general pattern of deposition is consistent with prevailing paleogeographic models calling for a northward, transverse growth of the proximal Brookian clastic wedge (for example, Bird and Molenaar, 1992).

Atigun syncline is a surprisingly isolated accumulation of coarse clastic sediment (fig. 1). The lack of similar facies to the east, in the Phillip Smith Mountains, is clearly related to deeper levels of erosion associated with Tertiary uplift (O’Sullivan and others, 1997). However, based on recent DGGS mapping west of Atigun syncline, the Fortress Mountain Formation consists of conglomerate-poor facies. The next significant accumulation of conglomerate is nearly $100 \mathrm{~km}$ west of Atigun syncline (fig. 1). The isolated nature of thick conglomeratic facies cannot be wholly explained as an artifact of erosional or structural effects. Instead, it seems more likely that the limited map extent of this facies is a primary depositional feature (also suggested by Mull, 1985) reflecting the localized development of widely spaced fan deltas. Similar to alluvial fans, most fan deltas are isolated systems with limited influence on neighboring environments (Orton and Reading, 1993). It is tempting to interpret the thick accumulation at Atigun syncline as the depositional record of a major mid-Cretaceous trunk drainage. Vincent and Elliott (1997) demonstrated that major paleovalleys in the Spanish Pyrenees persisted through long periods of mountain belt evolution. Further analogies and insight can be drawn from the North American Cordillera, where Lawton and others (1994) interpreted localized accumulations of gravel as the record of long-lived, antecedent drainages controlled by transverse structural zones. 


\section{Fan Delta Depositional System}

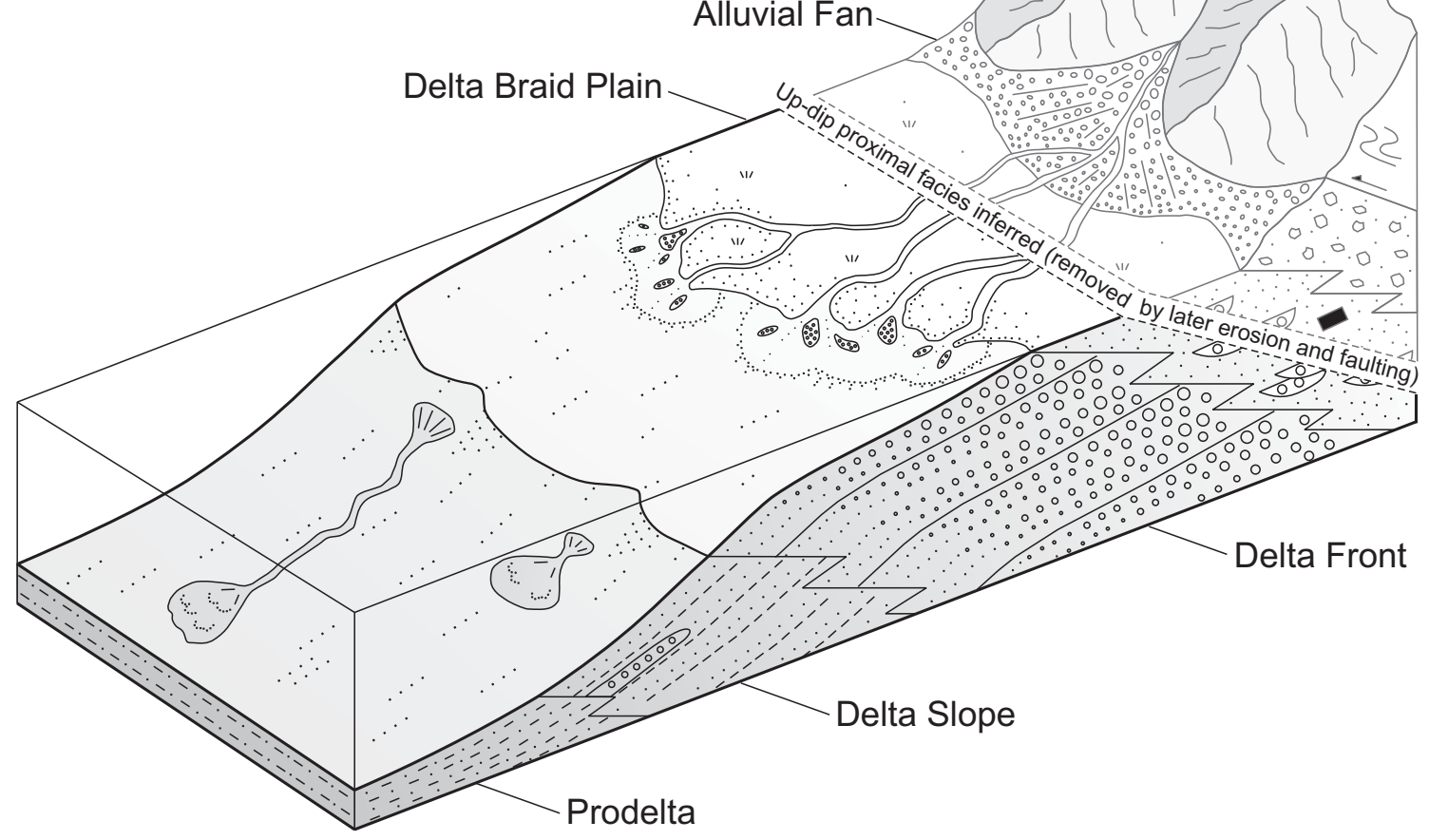

Figure 11. Summary block diagram illustrating the interpreted relationship of various facies components of the Atigun fan delta depositional system.

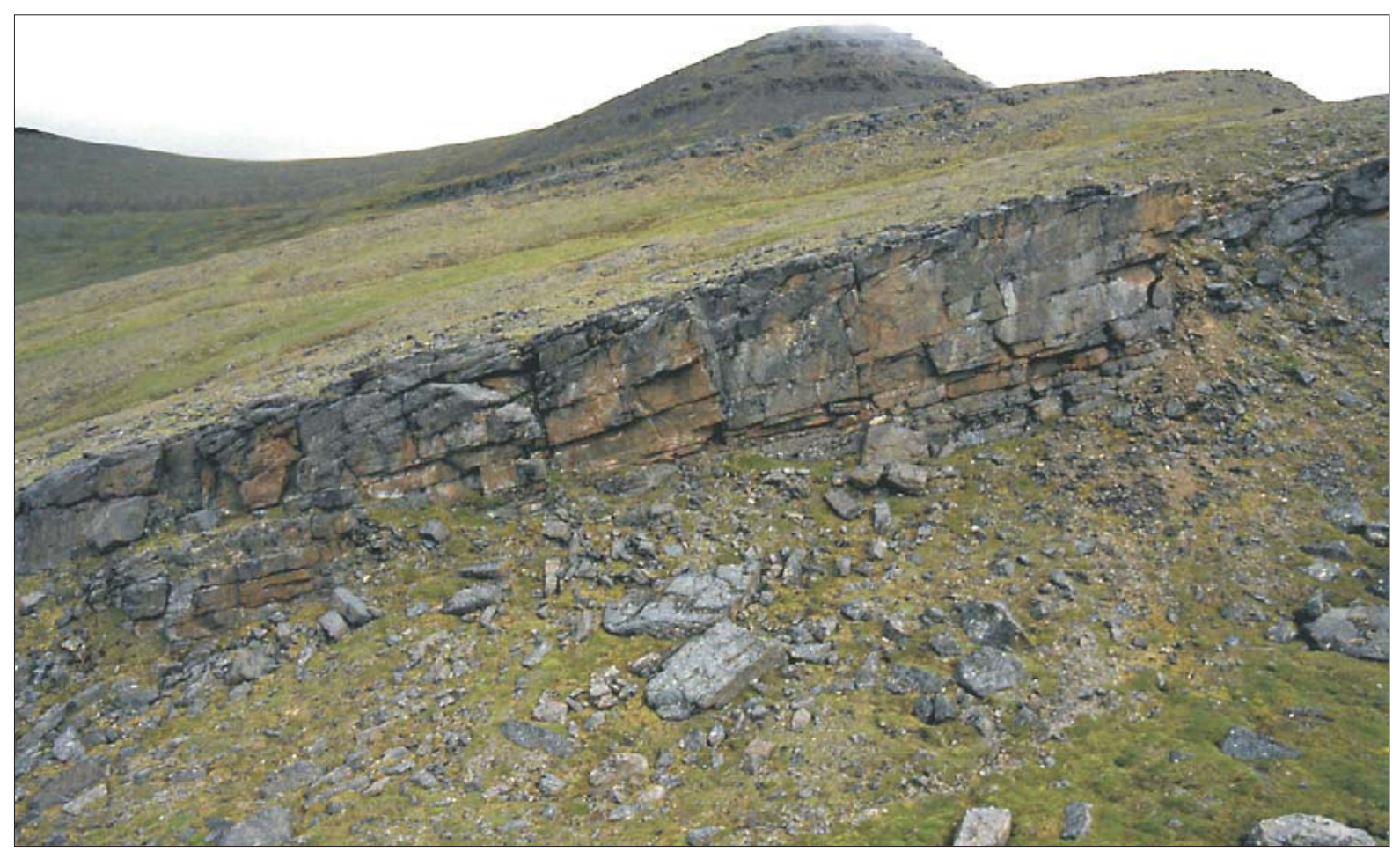

Figure 12. Prominent basinward-dipping shingled mouth bar foresets indicating northward (to the left) progradation of the fan delta system. Resistant cliff is approximately $15 \mathrm{~m}$ high. 


\section{AKNOWLEDGMENTS}

Support for fieldwork on the Fortress Mountain Formation was generously provided to the University of Wisconsin by Anadarko Petroleum Corporation. DGGS field studies on the North Slope are supported by industry contributions from Anadarko, FEX, BG Alaska, Chevron, Petro-Canada, Pioneer Natural Resources, ENI, Shell, and ConocoPhillips. Funding for DGGS geologic mapping in the Atigun area was provided by the state of Alaska and the U.S. Geological Survey STATEMAP program (award no. 01HQAG0054). A. Carroll, E. Parcher-Wartes, and S. Montayne are thanked for help in the field. This work benefited from numerous conversations with G. Mull, A. Carroll, D. LePain, J. Pietras, R. Reifenstuhl, E. Harris, D. Houseknecht, C. Schenk, and D. Hite. This paper benefitted from review comments by D. LePain.

\section{REFERENCES}

Bird, K.J., and Molenaar, C.M., 1992, The North Slope foreland basin, Alaska, in Macqueen, R.W., and Leckie, D.A., eds., Foreland basins and fold belts: AAPG Memoir no. 55, p. 363-393.

Bourgeois, J., and Leithold, E.L., 1984, Wave-worked conglomerates-depositional processes and criteria for recognition, in Koster, E.H., and Steel, R.J., eds., Sedimentology of gravels and conglomerates: Canadian Society of Petroleum Geologists, Memoir 10, p. 331-344.

Brosgé, W.P., Reiser, H.N., Dutro, J.T., Jr., and Detterman, R.L., 1979, Bedrock geologic map of the Phillip Smith Mountains quadrangle, Alaska: U.S. Geological Survey Miscellaneous Field Investigations Map MF 879-B, 2 sheets, scale 1:250,000.

Clifton, H.E., 1973, Pebble segregation and bed lenticularity in wave-worked versus alluvial gravel: Sedimentology, v. 20, p. 173-187.

Cole, F.E., Bird, K.J., Toro, J., Roure, F., O’Sullivan, P.B., Pawlewicz, M.J., and Howell, D.G., 1997, An integrated model for the frontal Brooks Range and Colville Basin 250 km west of the Trans-Alaska Crustal Transect: Journal of Geophysical Research, v. 102, p. 20,685-20,708.

Crowder, R.K., 1987, Cretaceous basin to shelf transition in northern Alaska; deposition of the Fortress Mountain Formation, in Tailleur, I.L., and Weimer, P., eds., Alaskan North Slope geology: Pacific Section, SEPM, Bakersfield, CA, v. 50, p. 449-458.

1989, Deposition of the Fortress Mountain Formation, in Mull, C.G., and Adams, K.E., eds., Dalton Highway, Yukon River to Prudhoe Bay, Alaska: Alaska Division of Geological \& Geophysical Surveys Guidebook 7, v. 2, p. 293-301.

Goff, R.A., 1990, Stratigraphy and sedimentology of the Fortress Mountain Formation at Atigun syncline, northern Alaska: Fairbanks, Alaska, University of Alaska Fairbanks, M.S. Thesis, 122 p.

Harris, E.E., Mull, C.G., Montayne, S., and Reifenstuhl, R.R., 2002, Geologic map of the Dalton Highway (Atigun Gorge to Slope Mountain) area, southeastern Arctic Foothills, Alaska: Alaska Division of Geological \& Geophysical Surveys Preliminary Interpretive Report 2002-2, 1 sheet, scale 1:63,360.

Hart, B.S., and Plint, A.G., 2003, Stratigraphy and sedimentology of shoreface and fluvial conglomerates; Insights from the Cardium Formation in Northwest Alberta and adjacent British Columbia: Bulletin of Canadian Petroleum Geology, v. 51, no. 4, p. 437-464.

Horton, B.K., and Schmitt, J.G., 1996, Sedimentology of the lacustrine fan-delta system, Miocene Horse Camp Formation, Nevada, USA: Sedimentology v. 43, p. 133-155.

Hunter, R.E., and Fox, J.E., 1976, Interpretation of depositional environments in the Fortress Mountain Formation, central Arctic Slope: United States Geological Survey Circular 733, p. 30-31.

Kleinspehn, K.L., Steel, R.J., Johannessen, E., and Netland, A., 1984, Conglomeratic fan-delta sequences, Late Carboniferous-Early Permian, western Spitsbergen, in Koster, E.H., and Steel, R.J. eds., Sedimentology of gravels and conglomerates: Canadian Society of Petroleum Geologists, Memoir 10, p. 279-294.

Lawton, T.F., Boyer, S.E., and Schmitt, J.G., 1994, Influence of inherited taper on structural variability and conglomerate distribution, Cordilleran fold and thrust belt, western United States: Geology, v. 22, p. 339-342.

Lopez-Blanco, M., 1993, Stratigraphy and sedimentary development of the Sant Llorec del Munt fan-delta complex (Eocene, southern Pyrenean foreland basin, northeast Spain), in Frostick, L., and Steel, R.J., (eds.), Tectonic controls and signatures in sedimentary successions: International Association of Sedimentology, Special Publication 20, p. 67-89.

Massari, F., and Parea, G.C., 1988, Progradational gravel beach sequences in a moderate- to high-energy, microtidal marine environment: Sedimentology, v. 35, p. 881-913. 
Miall, A.D., 1978, Lithofacies types and vertical profile models in braided river deposits: A summary, in Miall, A.D., ed., Fluvial sedimentology: Canadian Society of Petroleum Geology Memoir 5, 859 p.

Mickey, M.B., and Haga, H., 1987, Jurassic-Neocomian biostratigraphy, North Slope, Alaska, in Tailleur, I.L., and Weimer, P., eds., Alaskan North Slope geology: Pacific Section, SEPM, Bakersfield, CA, v. 50, p. 397-404.

Molenaar, C.M., Egbert, R.M., and Krystinik, L.F., 1988, Depositional facies, petrography, and reservoir potential of the Fortress Mountain Formation (Lower Cretaceous), central North Slope, Alaska, in Gryc, G., ed., Geology and exploration of the National Petroleum Reserve in Alaska, 1974-1982: U.S. Geological Survey Professional Paper 1399, p. 257-280.

Mull, C.G., 1985, Cretaceous tectonics, depositional cycles, and the Nanushuk Group, Brooks Range and Arctic Slope, Alaska, in Huffman, A.C., ed., Geology of the Nanushuk Group and related rocks, North Slope, Alaska: U.S. Geological Survey Bulletin 1614, p. 7-36.

Mull, C.G., and Harris, E.E., 1989, Road log from Chandalar Shelf (mile 237.1) to Prudhoe Bay (mile 414), in Mull, C.G., and Adams, K.E., eds., Dalton Highway, Yukon River to Prudhoe Bay, Alaska; Bedrock geology of the eastern Koyukuk Basin, central Brooks Range and eastcentral Arctic Slope: Alaska Division of Geological \& Geophysical Surveys Guidebook 7, vol. 1, p. 101-126.

Mull, C.G., Glenn, R.K., and Adams, K.E., 1997, Tectonic evolution of the central Brooks Range mountain front; Evidence from the Atigun Gorge region: Journal of Geophysical Research, v. 102, no. B9, p. 20,749-20,772.

Nemec, W., and Steel, R.J., 1984, Alluvial and coastal conglomerates; Their significant features and some comments on gravelly mass-flow deposits, in Koster, E.H., and Steel, R.J., eds., Sedimentology of gravels and conglomerates: Canadian Society of Petroleum Geologists Memoir 10, p. 1-31.

1988, What is a fan delta and how do we recognize it?, in Nemec, W., and Steel, R.J., eds., Fan deltas; Sedimentology and tectonic settings: London, Blackie and Son, p. 3-13.

Nemec, W., Steel, R.J., Porebski, S.J., and Spinnangr, A., 1984, Domba Conglomerate, Devonian, Norway; Process and lateral variability in a mass flow-dominated, lacustrine fan-delta, in Koster, E.H., and Steel, R.J., eds., Sedimentology of gravels and conglomerates: Canadian Society of Petroleum Geologists Memoir 10, p. 295-320.

Orton, G.J., and Reading, H.G., 1993, Variability of deltaic processes in terms of sediment supply, with particular emphasis on grain size: Sedimentology, v. 40, p. 475-512.

Patton, W.W., and Tailleur, I.L., 1964, Geology of the Killik-Itkillik region, Alaska: U.S. Geological Survey Professional Paper 303G, p. 409-500.

Postma, G., 1984, Slumps and their deposits on fan delta front and slope: Geology, v. 12, p. 27-30.

Rasmussen, H., 2000, Nearshore and alluvial facies in the Sant Llorenc del Munt depositional system; Recognition and development: Sedimentary Geology, v. 138, p. 71-98.

Ryherd, T.J., 1990, Fan-delta deposition in the Cretaceous (Albian) Fortress Mountain Formation, central North Slope, Alaska [abs.]: AAPG Bulletin, v. 74, p. 753.

Schenk, C.J., and Bird, K.J., 1992, Depositional sequences in Lower Cretaceous rocks, Atigun syncline and Slope Mountain areas, Alaska North Slope, in Dusel-Bacon, C., and Till, A.B., eds., Geologic Studies in Alaska by the U.S. Geological Survey, 1992: U.S. Geological Survey Bulletin 2068, p. 48-58.

Vincent, S.J., and Elliott, T., 1997, Long-lived transfer-zone paleovalleys in mountain belts; An example from the Tertiary of the Spanish Pyrenees: Journal of Sedimentary Research, v. 67, no. 2, p. 303310.

O'Sullivan, P.B., Murphy, J.M., and Blythe, A.E., 1997, Late Mesozoic and Cenozoic thermotectonic evolution of the central Brooks Range and adjacent North Slope foreland basin, Alaska-including fission track results from the Trans-Alaska Crustal Transect (TACT): Journal of Geophysical Research, v. 102, p. 20,821-20,845.

Wartes, M.A., and Carroll, A.R., 2002, Detailed facies analysis of the Lower Cretaceous Fortress Mountain Formation, Atigun syncline, northern Alaska [abs.]: American Association of Petroleum Geologists, Pacific Section Meeting Abstracts, AAPG Bulletin, v. 86, no. 6, p. 1,162.

Wescott, W.A., and Ethridge, F.G., 1980, Fan-delta sedimentology and tectonic setting-Yallahs fan-delta, southeast Jamaica: American Association of Petroleum Geologists Bulletin, v. 64, p. 374-399. 